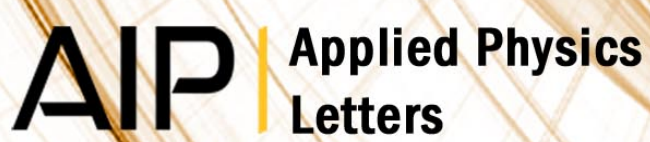

\section{Thermal emission from large area chemical vapor deposited graphene devices}

I. J. Luxmoore, C. Adlem, T. Poole, L. M. Lawton, N. H. Mahlmeister et al.

Citation: Appl. Phys. Lett. 103, 131906 (2013); doi: 10.1063/1.4821939

View online: http://dx.doi.org/10.1063/1.4821939

View Table of Contents: http://apl.aip.org/resource/1/APPLAB/v103/i13

Published by the AIP Publishing LLC.

Additional information on Appl. Phys. Lett.

Journal Homepage: http://apl.aip.org/

Journal Information: http://apl.aip.org/about/about_the_journal

Top downloads: http://apl.aip.org/features/most_downloaded

Information for Authors: http://apl.aip.org/authors

\section{ADVERTISEMENT}

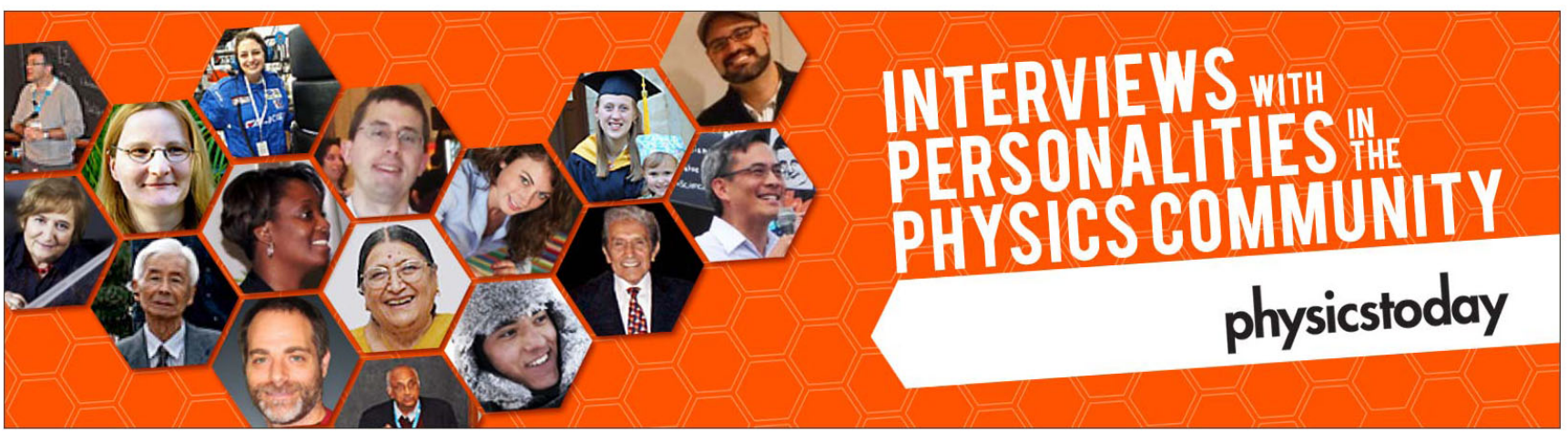




\title{
Thermal emission from large area chemical vapor deposited graphene devices
}

\author{
I. J. Luxmoore, ${ }^{\text {a) }}$ C. Adlem, T. Poole, L. M. Lawton, N. H. Mahlmeister, and G. R. Nash \\ College of Engineering, Mathematics, and Physical Sciences, University of Exeter, Exeter EX4 4QF, \\ United Kingdom
}

(Received 30 July 2013; accepted 6 September 2013; published online 23 September 2013)

\begin{abstract}
The spatial variation of thermal emission from large area graphene grown by chemical vapor deposition, transferred onto $\mathrm{SiO}_{2} / \mathrm{Si}$ substrates and fabricated into field effect transistor structures, has been investigated using infra-red microscopy. A peak in thermal emission occurs, the position of which can be altered by reversal of the current direction. The experimental results are compared with a one dimensional finite element model, which accounts for Joule heating and electrostatic effects, and it is found that the thermal emission is governed by the charge distribution in the graphene and maximum Joule heating occurs at the point of minimum charge density. (C) 2013 AIP Publishing LLC. [http://dx.doi.org/10.1063/1.4821939]
\end{abstract}

Thanks to its high intrinsic mobility and thermal conductivity, graphene has great potential for fundamental scientific research and in a multitude of diverse electronic and optoelectronic applications, such as high frequency integrated circuits, ${ }^{1}$ transparent-flexible electronics, ${ }^{2}$ and in $\mathrm{THz}$ components. ${ }^{3,4}$ Graphene, grown by chemical vapor deposition (CVD) on copper ${ }^{5}$ and transferred to insulating substrates for device applications, ${ }^{6,7}$ has the advantage of large area coverage and the potential for scalable device architectures, but suffers from lower mobility and thermal conductivity when compared with exfoliated graphene. ${ }^{8-10}$ Nevertheless, the material quality is sufficient for many device applications ${ }^{1,2,4}$ and it is important that the electrical and thermal properties are well understood. Over the last few years, thermal emission from graphene has been found to be a useful probe of the electronic properties of graphene and has been used to investigate charge transport in small exfoliated graphene samples both in the diffusive ${ }^{11-14}$ and ballistic $^{15}$ transport regimes. In the high (electrical) field regime, transport in the graphene channel is ambipolar and the position of the charge neutrality point can be determined from the point of maximum thermal emission using infrared (IR) scanning microscopy. ${ }^{11-13}$

In this work, we have investigated the thermal emission at high field from technologically important, large area CVD graphene devices. Using IR scanning microscopy we have measured the spatial variation of the thermal emission from $3 \mathrm{~mm} \times 3 \mathrm{~mm}$ devices under a range of drive conditions. The experimental results are compared with a 1D finite element model, which accounts for Joule heating and electrostatic effects $^{11}$ and it is found that the thermal emission is governed by the charge distribution in the graphene and that maximum Joule heating occurs at the point of minimum charge density. Previous thermal emission experiments ${ }^{11-13}$ have concentrated on relatively small exfoliated graphene devices with widths of around $\sim 5 \mu \mathrm{m}$ and lengths up to $\sim 50 \mu \mathrm{m}$, driven with a constant bias. In our experiments we

\footnotetext{
${ }^{\text {a) }}$ Author to whom correspondence should be addressed. Electronic mail: i.j.luxmoore@exeter.ac.uk
}

investigate very large area CVD graphene devices driven with a pulsed current source; despite the experimental differences we observe qualitatively similar behavior, resulting from the charge distribution in the graphene channel. However, although the current densities are significantly smaller in our case, we observe a large temperature increase, which we attribute to poor overall thermal contact between the graphene and the $\mathrm{SiO}_{2}$ substrate and the thermal diffusion time through the substrate.

The devices were fabricated from pre-transferred monolayer graphene (Graphene Supermarket) on $300 \mathrm{~nm}$ thick $\mathrm{SiO}_{2}$, with the highly doped Si substrate used as a back gate. Large area devices were defined using electron beam lithography and an $\mathrm{O}_{2} / \mathrm{Ar}$ reactive ion etch to leave a $3.24 \mathrm{~mm} \times 3.24 \mathrm{~mm}$ area of graphene. $\mathrm{Cr} / \mathrm{Au}(5 / 50 \mathrm{~nm}), 3.6 \mathrm{~mm}$ long and $180 \mu \mathrm{m}$ wide, source and drain contacts were deposited on top of the graphene using thermal evaporation. Fig. 1(a) shows a schematic of the device. In this paper, we present results from a typical device. The monolayer nature of the graphene was confirmed using Raman spectroscopy with a $100 \mathrm{~mW} 532 \mathrm{~nm}$ continuous wave laser. In order to verify the uniformity of the graphene, multiple spectra were recorded across the sample with very little variation between measurements. The average of these spectra is presented in Fig. 1(b). The 2D and G peak are observed at $2885 \mathrm{~cm}^{-1}$ and $1590 \mathrm{~cm}^{-1}$, which is a characteristic of monolayer graphene. ${ }^{16}$ The D peak at $1346 \mathrm{~cm}^{-1}$, which can be attributed to the defects caused by unintentional doping and wrinkles formed during the transfer process, is small and suggests that the overall quality of the transferred graphene is high.

The devices were mounted on ceramic chip holders and placed in a vacuum chamber, with a $\mathrm{CaF}_{2}$ window for optical access, which was evacuated to $\sim 10^{5}$ millibars. Thermal emission measurements were performed using a Keithley 6221 current source with devices driven by a $1 \mathrm{kHz}$ square waveform (50\% duty cycle), at peak injection currents up to $20 \mathrm{~mA}$. The thermal emission was collected using a $15 \mathrm{X}$ reflecting objective lens $(\mathrm{NA}=0.28)$ and focused, using a $\mathrm{CaF}_{2}$ lens, onto a liquid nitrogen cooled $\mathrm{MgCdTe}$ detector, with a $2-12 \mu \mathrm{m}$ response. The reflecting objective, $\mathrm{CaF}_{2}$ lens, 

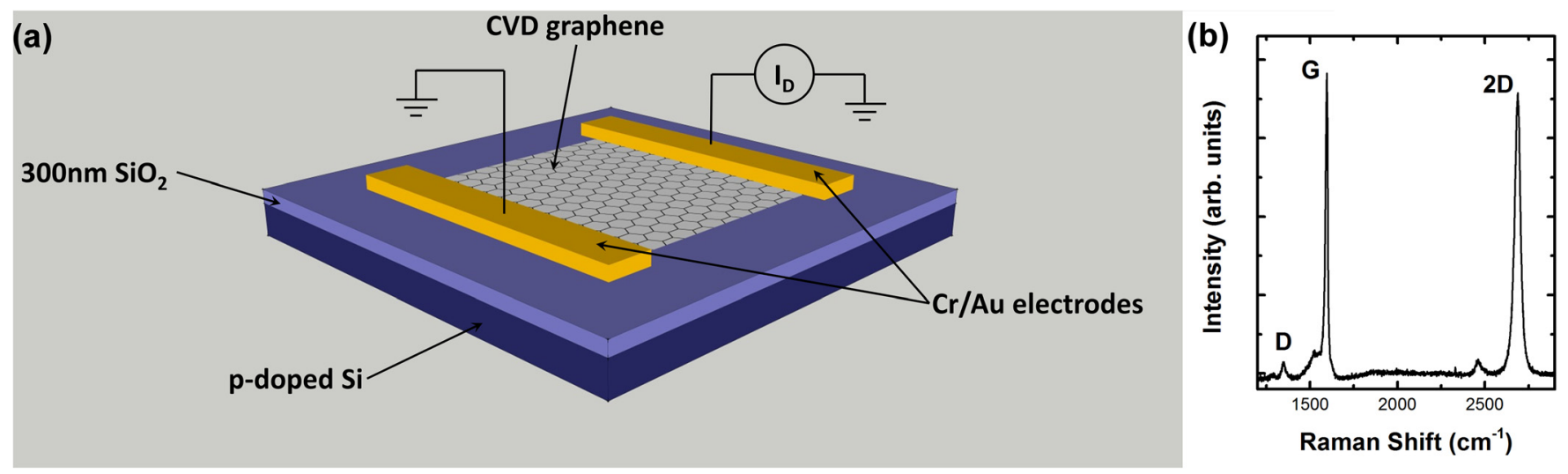

FIG. 1. (a) Schematic diagram of the large area CVD graphene field effect transistor. (b) Average Raman spectrum recorded from the monolayer graphene.

and detector were mounted on a xy-stage and the spatial variation of the thermal emission was measured by scanning the microscope system over the sample. The signal from the detector was amplified by a low noise preamplifier, and passed to a lock-in amplifier for phase sensitive measurement.

To characterize the electronic properties of the monolayer graphene, field effect measurements were employed. A small (100 nA) alternating current, $\mathrm{I}_{\mathrm{D}}$ was applied to the device and the voltage dropped across the device recorded as a function of the voltage applied to the back gate, $\mathrm{V}_{\mathrm{G}}$. The field effect characteristic is shown in Fig. 2, for forward and reverse gate voltage sweeps. Even for this very large device, there is a peak in the resistance typical of graphene samples. The field effect characteristic displays hysteretic behavior, which is an indicator of charge transfer from surface contaminants and/or charge trapping in the dielectric substrate. ${ }^{17,18}$ The experimental data was fitted in the voltage range close to the Dirac point with a phenomenological model, ${ }^{19}$ which accounts for gate induced and thermally generated carriers and "puddle" carriers ${ }^{20,21}$ resulting from spatial charge variations. This fitting allowed values to be extracted for the mobility, $\mu=1100 \mathrm{~cm}^{2} \mathrm{~V}^{-1} \mathrm{~s}^{-1}$, puddle carrier density, $\mathrm{n}_{\mathrm{pd}}=7.5 \times 10^{11} \mathrm{~cm}^{-2}$, and Dirac voltage, $\mathrm{V}_{0}=17.7 \mathrm{~V}$ for the positive gate sweep and $\mu=1190 \mathrm{~cm}^{2} \mathrm{~V}^{-1} \mathrm{~s}^{-1}, \mathrm{n}_{\mathrm{pd}}=7 \times 10^{11} \mathrm{~cm}^{-2}$, and $\mathrm{V}_{0}=28.6 \mathrm{~V}$

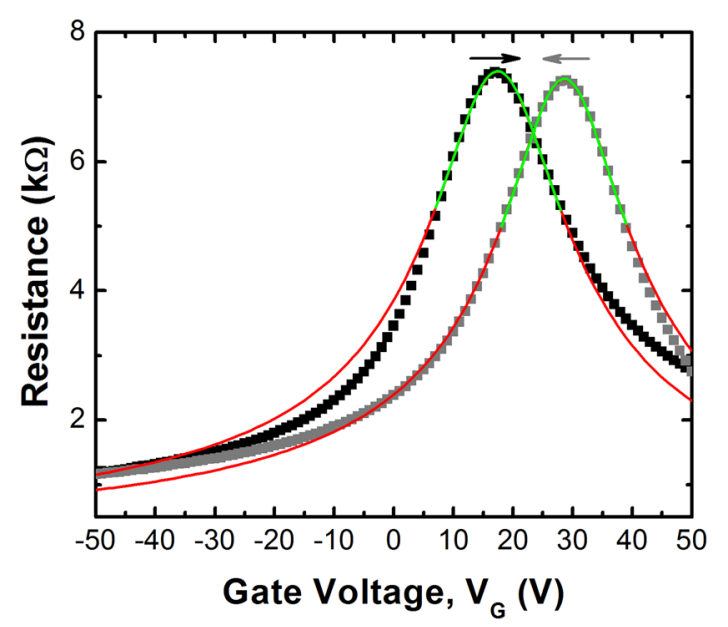

FIG. 2. Field effect measurement of the $3 \mathrm{~mm} \times 3 \mathrm{~mm}$ CVD graphene device. The black (grey) data points show the positive (negative) gate sweep. The green line show fits to the data close to $V_{0}$ and the red lines show extrapolations of these fits to high bias. for the negative gate sweep. The data deviates from the simple model, at higher fields, due to a carrier density dependent mobility and suggests that the dominant scattering mechanism switches from Coulomb to phonon at higher carrier density. ${ }^{22}$ The characteristic also shows some asymmetry between hole and electron conduction, which is also symptomatic of surface contamination. ${ }^{18}$ As a result of the large overlap of the source and drain contacts with the graphene, the contact resistance is estimated to be less than $1 \%$ of the channel resistance. ${ }^{14}$ Therefore, Joule heating and thermal emission occurs predominantly in the graphene and not at the contacts. The contacts do, however, play a role in the spatial emission of the device, because they are thermally anchored to the ambient temperature and therefore act as heat sinks.

In Figures 3(a) and 3(b), the spatial variation of the thermal emission from the device, under zero applied gate bias $\left(V_{G}=0\right)$, is shown for forward bias drain currents of $20 \mathrm{~mA}$ and $10 \mathrm{~mA}$, respectively. Figures 3(c) and 3(d) show the same measurements, but with the direction of current reversed (drain currents of $-20 \mathrm{~mA}$ and $-10 \mathrm{~mA}$, respectively). In all cases, emission is only observed from the $3 \mathrm{~mm} \times 3 \mathrm{~mm}$ area corresponding to the graphene, indicating that the whole graphene area is heating up whereas the $\mathrm{SiO}_{2}$ is not. At a drain current of $20 \mathrm{~mA}$, the emission from the device is dominated by a single large hot-spot close to the drain contact (Fig. 3(a)). On reversing the current direction, the hot-spot switches to the opposite side of the graphene channel, as seen in Fig. 3(c). This behavior is similar to that observed in micron-scale exfoliated graphene samples, where the thermal emission is dominated by Joule heating governed by the charge distribution along the channel, ${ }^{11-13}$ but on a much larger scale. The magnitude of the emission at the hot-spot demonstrates an approximate dependence on the current squared (see inset to Fig. 3(e)), but at lower currents the hotspot becomes less well defined. For example, at $\pm 10 \mathrm{~mA}$ (Figures 3(b) and 3(c)), we observed numerous bright areas where the graphene temperature is elevated. This could be as a result of areas of higher resistivity, leading to increased Joule heating, or in a spatial variation of the thermal resistance between the graphene and the substrate.

To qualitatively explain the spatial emission, we use a 1D finite element model ${ }^{11}$ to calculate the charge density, electric field, and temperature along the graphene channel, where the charge density includes the effect of doping due to 

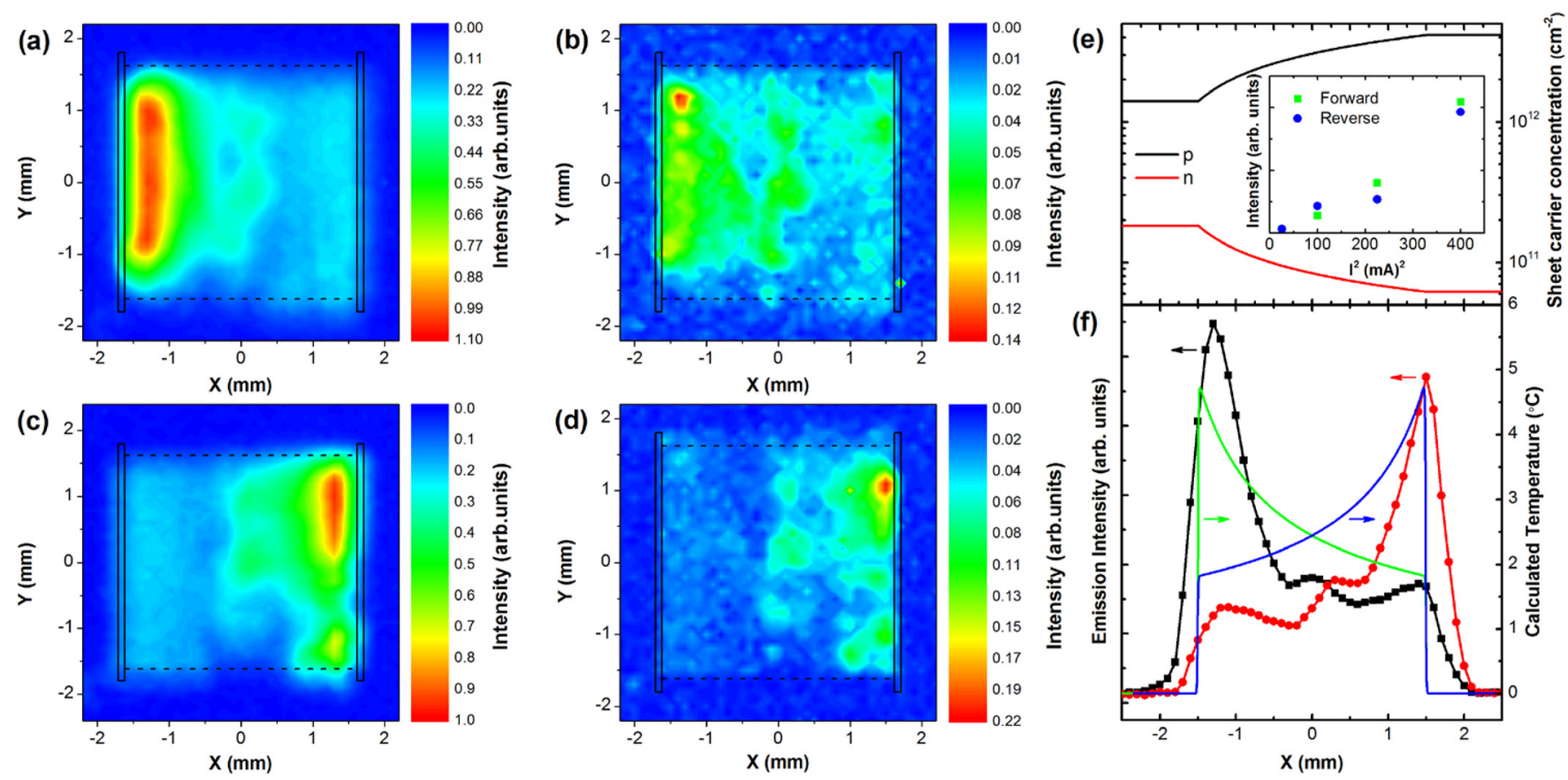

FIG. 3. Thermal emission mapped from a large area graphene device under forward bias (a) $I_{D}=20 \mathrm{~mA}$ and (b) $I_{D}=10 \mathrm{~mA}$, and reverse bias (c) $I_{D}=-20 \mathrm{~mA}$ and (d) $\mathrm{I}_{\mathrm{D}}=-10 \mathrm{~mA}$. In (a)-(d) the dashed line indicates the position of the monolayer graphene and the solid line the position of the Cr/Au contacts. (e) Sheet carrier concentration along the channel of the device, calculated using a 1D finite element model. The inset shows a plot of the emission intensity as a function of the drive current squared. (f) Average emission intensity and calculated temperature profile along the channel. The black (red) data points show the experimental emission data and the green (blue) lines show the calculated temperature profile for forward (reverse) bias.

traps in the $\mathrm{Si} / \mathrm{SiO}_{2}$, and where the temperature increase is assigned to Joule heating. The calculated values of carrier concentration and temperature are plotted in Figures 3(e) and 3(f), respectively, for a drain current of $+20 \mathrm{~mA}$, using the model parameters extracted from the field effect measurements. The results shown in Fig. 3(e) indicate the device is operating in the hole doped regime, with the hole concentration varying by a factor of $\sim 3$ between the source and drain ends of the channel. This reduced charge density close to the drain results in increased Joule heating at the drain end, with the calculated temperature profile for $\pm 20 \mathrm{~mA}$ plotted in Fig. 3(f), along with averaged thermal emission intensity profiles along the channel. As observed in the experiment, the simulations predict the presence of a hotspot at one end of the device that switches position when the direction of current is reversed. The qualitative agreement between experiment and simulation suggest that the observed non-uniform thermal emission is caused by the distinctive charge distribution in the graphene, and that the maximum Joule heating (and hence temperature) occurs at the point of minimum charge density.

To compare experiments and simulations quantitatively, the temperature of the sample during emission was estimated by assuming that the graphene behaved as a grey body and measuring the signal obtained after passing the emitted light through a number of optical filters. ${ }^{13}$ At $20 \mathrm{~mA}$ the sample reached a peak temperature of $\sim 370 \mathrm{~K}$, which is much higher than the few degree increase estimated by the simulations. In addition, the temperature is high when compared with previous measurements, ${ }^{11-13}$ given that the current density in our case is much lower. This high measured temperature and deviation from the predictions of the simulation most likely originates from the thermal coupling of the graphene to the
$\mathrm{SiO}_{2}$ substrate. In the simulations, it was assumed that the thermal conductance of the graphene- $\mathrm{SiO}_{2}$ boundary was $10^{8} \mathrm{Wm}^{-2} \mathrm{~K}^{-1}$, as measured for exfoliated graphene. ${ }^{23}$ However, in the case of CVD graphene transferred to $\mathrm{SiO}_{2}$ it is likely that this value may be much lower, as a large proportion of the graphene is likely to be suspended above the $\mathrm{SiO}_{2}$ due to wrinkles in the graphene. ${ }^{24}$ The model also assumes a steady-state operation, whereas our measurements are performed at a frequency of $1 \mathrm{kHz}$, with a $50 \%$ duty cycle. This timescale is similar to the thermal diffusion time through the Si wafer of $\sim 0.3 \mathrm{~ms},{ }^{25}$ suggesting that the graphene is not able to cool down in between current pulses. Further work is underway to investigate both the coupling of the graphene to the substrate and also the frequency dependence of the measurements.

In conclusion, we have investigated the spatial variation of thermal emission from large area CVD graphene devices. Using a 1D finite element model, which accounts for Joule heating and electrostatic effects, it is found that the nonuniform thermal emission is governed by the charge distribution in the graphene and that maximum Joule heating occurs at the point of minimum charge density. The observed thermal emission from these large area devices is therefore qualitatively the same as seen from much smaller exfoliated devices.

The authors would like to thank G. Hrkac and C. H. Gan for useful discussions. This research was supported by the Engineering and Physical Sciences Research Council, and the European Union under the FET-open grant GOSFEL.

${ }^{1}$ Y.-M. Lin, A. Valdes-Garcia, S.-J. Han, D. B. Farmer, I. Meric, Y. Sun, Y. Wu, C. Dimitrakopoulos, A. Grill, P. Avouris, and K. A. Jenkins, Science 332, 1294 (2011). 
${ }^{2}$ S. Bae, H. Kim, Y. Lee, X. Xu, J.-S. Park, Y. Zheng, J. Balakrishnan, T. Lei, H. R. Kim, Y. Il Song, Y.-J. Kim, K. S. Kim, B. Ozyilmaz, J.-H. Ahn, B. H. Hong, and S. Iijima, Nat. Nanotechnol. 5, 574 (2010).

${ }^{3}$ L. Vicarelli, M. S. Vitiello, D. Coquillat, A. Lombardo, A. C. Ferrari, W. Knap, M. Polini, V. Pellegrini, and A. Tredicucci, Nature Mater. 11, 865 (2012).

${ }^{4}$ S. H. Lee, M. Choi, T.-T. Kim, S. Lee, M. Liu, X. Yin, H. K. Choi, S. S. Lee, C.-G. Choi, S.-Y. Choi, X. Zhang, and B. Min, Nature Mater. 11, 936 (2012).

${ }^{5}$ X. Li, W. Cai, J. An, S. Kim, J. Nah, D. Yang, R. Piner, A. Velamakanni, I. Jung, E. Tutuc, S. K. Banerjee, L. Colombo, and R. S. Ruoff, Science 324, 1312 (2009).

${ }^{6}$ X. Li, Y. Zhu, W. Cai, M. Borysiak, B. Han, D. Chen, R. D. Piner, L. Colombo, and R. S. Ruoff, Nano Lett. 9, 4359 (2009).

${ }^{7}$ X. Liang, B. A. Sperling, I. Calizo, G. Cheng, C. A. Hacker, Q. Zhang, Y. Obeng, K. Yan, H. Peng, Q. Li, X. Zhu, H. Yuan, A. R. H. Walker, Z. Liu, L.-M. Peng, and C. A. Richter, ACS Nano 5, 9144 (2011).

${ }^{8}$ W. Cai, A. L. Moore, Y. Zhu, X. Li, S. Chen, L. Shi, and R. S. Ruoff, Nano Lett. 10, 1645 (2010).

${ }^{9}$ A. A. Balandin, Nature Mater. 10, 569 (2011).

${ }^{10}$ D. L. Nika and A. A. Balandin, J. Phys.: Condens. Matter 24, 233203 (2012).

${ }^{11}$ M. Bae, Z. Ong, D. Estrada, and E. Pop, Nano Lett. 10, 4787 (2010).

${ }^{12}$ M.-H. Bae, S. Islam, V. E. Dorgan, and E. Pop, ACS Nano 5, 7936 (2011).
${ }^{13}$ M. Freitag, H.-Y. Chiu, M. Steiner, V. Perebeinos, and P. Avouris, Nat. Nanotechnol. 5, 497 (2010).

${ }^{14}$ K. L. Grosse, M.-H. Bae, F. Lian, E. Pop, and W. P. King, Nat. Nanotechnol. 6, 287 (2011).

${ }^{15}$ M.-H. Bae, Z. Li, Z. Aksamija, P. N. Martin, F. Xiong, Z.-Y. Ong, I. Knezevic, and E. Pop, Nat. Commun. 4, 1734 (2013).

${ }^{16}$ A. C. Ferrari, J. C. Meyer, V. Scardaci, C. Casiraghi, M. Lazzeri, F. Mauri, S. Piscanec, D. Jiang, K. S. Novoselov, S. Roth, and A. K. Geim, Phys. Rev. Lett. 97, 187401 (2006).

${ }^{17}$ H. Wang, Y. Wu, C. Cong, J. Shang, and T. Yu, ACS Nano 4, 7221 (2010).

${ }^{18}$ T. Lohmann, K. von Klitzing, and J. H. Smet, Nano Lett. 9, 1973 (2009).

${ }^{19}$ V. E. Dorgan, M.-H. Bae, and E. Pop, Appl. Phys. Lett. 97, 082112 (2010).

${ }^{20}$ A. Deshpande, W. Bao, F. Miao, C. Lau, and B. LeRoy, Phys. Rev. B 79, 205411 (2009).

${ }^{21}$ J. Martin, N. Akerman, G. Ulbricht, T. Lohmann, J. H. Smet, K. von Klitzing, and A. Yacoby, Nat. Phys. 4, 144 (2008).

${ }^{22}$ W. Zhu, V. Perebeinos, M. Freitag, and P. Avouris, Phys. Rev. B 80, 235402 (2009).

${ }^{23}$ Z. Chen, W. Jang, W. Bao, C. N. Lau, and C. Dames, Appl. Phys. Lett. 95, 161910 (2009).

${ }^{24}$ N. Liu, Z. Pan, L. Fu, C. Zhang, B. Dai, and Z. Liu, Nano Res. 4, 996 (2011).

${ }^{25}$ E. Pop, Nano Res. 3, 147 (2010). 